\title{
Impactos decorrentes da síndrome de burnout nos profissionais do Serviço de Atendimento Móvel de Urgência (SAMU)
}

\author{
Impacts of burnout syndrome on the professionals of the Mobile Emergency Assistance \\ Service (SAMU)
}
Impactos del síndrome de agotamiento em los profesionales del Servicio Móvil de Asistencia de Emergencia (SAMU)

\begin{abstract}
Francisca Maria Pereira da Cruz ${ }^{1}$, Andréia da Silva Nascimento Pontes ${ }^{1}$, Tatiana Naiana Rodrigues dos Santos Porto ${ }^{1}$, Gaubeline Teixeira Feitosa ${ }^{1}$, Benedito Pereira de Sousa Neto ${ }^{1}$, Nilton Andrade Magalhães ${ }^{1}$, Vinícius de Sousa Martins ${ }^{1}$, Dorivaldo Pereira Carvalho ${ }^{1}$, Érica Natasha Duarte Silva ${ }^{1}$, Luciana Stanford Baldoino ${ }^{1 *}$.
\end{abstract}

\section{RESUMO}

Objetivo: Verificar os impactos decorrentes da síndrome de burnout nos profissionais de um Serviço de Atendimento Móvel de Urgência (SAMU). Métodos: Trata-se de uma pesquisa descritiva, exploratória de abordagem quantitativa. A pesquisa foi realizada em uma instituição pública que realiza atendimentos de urgência emergência, com 43 profissionais que exercem alguma função no serviço de atendimento móvel de urgência. Resultados: Percebe-se que $26(60,5 \%)$ dos profissionais eram do sexo masculino, com a faixa etária entre 31 a 50 anos $32(74,5 \%)$, o estado civil da maioria corresponde $21(48,8 \%)$ aos casados, sendo que $22(51,2 \%)$ tem de 2 a 3 filhos. No que diz respeito à função exercida na instituição, a que mais prevaleceu foi a de técnica em enfermagem e motorista; ambos com 14 (32,6\%). No que se refere à dimensão exaustão emocional $58,5 \%$ responderam nunca sentirem emocionalmente exausto no trabalho. Quanto à despersonalização destacam-se os $61,0 \%$ que mencionaram sempre se sentirem cheios de alegrias e $62,5 \%$ sempre entendem com facilidades os sentimentos dos pacientes atendidos. Conclusão: Esta pesquisa permitiu identificar os fatores nos profissionais que predominam para somatória da síndrome de burnout.

Palavras-chave: Síndrome de burnout, Exaustão profissional, Trabalho.

\begin{abstract}
Objective: To verify the impacts of the burnout syndrome on the professionals of a Mobile Emergency Service (SAMU). Methods: This is a descriptive, exploratory research with a quantitative approach. The research was conducted in a public institution that provides emergency care, with 43 professionals who perform some function in the mobile emergency service. Results: It is noticed that $26(60.5 \%)$ of the professionals were male, with the age group between 31 to 50 years old $32(74.5 \%)$, the marital status of the majority corresponds to $21(48.8 \%)$ married people, $22(51.2 \%)$ having 2 to 3 children. With regard to the role exercised in the institution, the one that prevailed most was that of nursing technician and driver; both with 14 (32.6\%). Regarding the emotional exhaustion dimension, 58.5\% answered that they never felt emotionally exhausted at work. Regarding depersonalization, $61.0 \%$ stand out, who mentioned always feeling full of joy and $62.5 \%$ always easily understand the feelings of the patients attended. Conclusion: This research allowed to identify the factors in the professionals that predominate for the sum of the burnout syndrome.
\end{abstract}

Keywords: Burnout syndrome, Professional exhaustion, Work.

${ }^{1}$ Faculdade de Ensino Superior Múltiplo-IESM, Timon - MA. *E-mail: Isbaldoino@hotmail.com 


\section{RESUMEN}

Objetivo: Verificar los impactos del síndrome de burnout en los profesionales de un Servicio de Emergencia Móvil (SAMU). Métodos: Esta es una investigación exploratoria descriptiva con un enfoque cuantitativo. La investigación se realizó en una institución pública que brinda atención de emergencia, con 43 profesionales que realizan alguna función en el servicio de emergencia móvil. Resultados: se puede observar que 26 $(60.5 \%)$ de los profesionales eran hombres, con un grupo de edad entre 31 y 50 años 32 ( $74.5 \%)$, el estado civil de la mayoría corresponde a 21 (48.8\%) personas casadas, 22 (51.2\%) con 2 a 3 hijos. Con respecto al papel ejercido en la institución, el que más prevaleció fue el de técnico de enfermería y conductor; ambos con $14(32,6 \%)$. Con respecto a la dimensión del agotamiento emocional, el 58.5\% respondió que nunca se sintió emocionalmente agotado en el trabajo. Con respecto a la despersonalización, se destaca el $61.0 \%$, que mencionó sentirse siempre lleno de alegría y el $62.5 \%$ siempre entiende fácilmente los sentimientos de los pacientes atendidos. Conclusión: Esta investigación permitió identificar los factores en los profesionales que predominan para la suma del síndrome de burnout.

Palabras clave: Síndrome de burnout, Agotamiento professional, Trabajo.

\section{INTRODUÇÃO}

A Síndrome de Burnout (SB) é considerada uma reação ao estresse ocupacional crônico no trabalho e as reações físicas e emocionais prejudicadas que ocorrem (MORAIS AJD, et al., 2018). Nesse sentido, a Síndrome de Burnout (SB) ou esgotamento profissional, é classificada como uma das consequências relacionada ao estresse profissional na saúde é considerada uma doença do trabalho e tem-se tornado um problema de saúde pública (FERNANDES LS, et al., 2018).

Nessa nova configuração organizacional, são requeridas novas exigências de qualificação e de competências no trabalho e do profissional. Como resultado, novas enfermidades surgem em decorrência dessas mudanças. Os agravos mentais, físicos e emocionais ocupam lugar de destaque. Dentre eles encontra-se a Síndrome de Burnout (PÊGO FPL e PEGO DR, 2016; MOTA GS, et al., 2016).

As mudanças que têm ocorrido no mundo do trabalho, como, por exemplo, o processo de globalização da economia, as novas tecnologias, a grande competição no mercado de trabalho, a necessidade de se produzir mais e mais rápido evitando desperdícios, entre muitos outros fatores, acabam gerando um desgaste físico e emocional nos trabalhadores (TAVARES JAB, et al., 2017).

As cidades de São Paulo e Porto Alegre estão entre as que apresenta mais pessoas prejudicadas na faixa etária de 25 a 60 anos, revela que no Brasil o problema atinge cerca de $30 \%$ da população economicamente ativa (PEA) (VASCONCELOS EM, et al., 2016). Mostram ainda que, 94\% se sentem incapacitados para trabalhar, $89 \%$ praticam presenteísmo e $47 \%$ sofrem de depressão. Tem-se mais incidência em mulheres com 40 anos de idade, dupla jornada feminina, trabalho e afazeres domésticos, contribui diretamente para a percepção maior da síndrome no sexo feminino, demonstrando que ainda hoje as mulheres são mais pressionadas por questão biológicas e sociais (SILVA RAD, et al., 2018).

Evidencia-se, que a prevalência da síndrome é mais perceptível em trabalhadores jovens que ainda não alcançaram seus 30 anos, os fatores como a falta de experiência, que acarreta insegurança ou choque de realidade, o medo do desemprego e o desgaste físico e psicológico, que muitas vezes é ignorado, assim como a inexistência do salário contribui para o reconhecimento da SB nesse público (PAIVA JDM, et al., 2019; SILVA CCS, et al., 2017).

Vale salientar que a legislação Brasileira no ano de 1999 considera a SB um transtorno mental pertencente ao grupo V da CID-10, definição feita por decreto lei sob o no 3048/99. Sua caracterização está descrita no manual de procedimentos para o serviço de saúde, e tendo assim seu diagnóstico constatado abre-se a possibilidade 18 de tratamento, afastamento do trabalho e compensação financeira (DUTRA FC, et al., 2016; SOUSA GJ e ROSSI EJR, 2016). 
A Síndrome de Burnout tem sido considerada uma importante questão de saúde pública, o ambiente do profissional de saúde hoje no Brasil contribui para os agravos ocupacionais do caráter psicossocial dos profissionais. No entanto, a SB é um sério processo de deterioro da qualidade de vida do trabalhador, tendo em vista suas graves implicações para a saúde física e mental (SOUSA AMJ, et al., 2018; OLIVEIRA LPS e ARAÚJO GF, 2016; NOGUEIRA LS, et al., 2018).

Os autores afirmam que a importância de agentes estressores psicossomáticos é hoje amplamente reconhecida, sendo tão potentes quanto os microrganismos ou a insalubridade, no desencadeamento das doenças. A sintomatologia da SB se apresenta mediante expressões físicas, comportamentais e psíquicas; não necessariamente, entretanto, todos os sintomas simultaneamente. Assim a sintomatologia da SB, depressiva são pontos relevantes de reflexão e da elaboração de programas de saúde ocupacional para o tratamento dos agentes estressores (SOUSA MKP, et al., 2019; BATISTA LMA, et al., 2016; SARTORETO IS e KURGGANT P, 2017).

De um modo geral, a literatura aponta que o tratamento para o burnout é realizado por meio de psicoterapia que, de acordo com o caso, pode solicitar o uso de medicações, caso a pessoa apresente problemas biofisiológicos, tais como: dores, alergias, alteração na pressão arterial, problemas cardíacos, insônia, e outras possíveis intercorrências. No que se refere à medicação, então, esta pode ser relacionada a analgésicos, complementos minerais, ansiolíticos e antidepressivos, de acordo com cada sintomatologia apresentada pelo indivíduo acometido com o burnout (SILVA CCS, et al., 2017; SILVA RNS, et al., 2015).

A partir desse enfoque, o estudo teve como objetivo verificar os impactos decorrentes da síndrome de burnout nos profissionais de um Serviço de Atendimento Móvel de Urgência - SAMU em um município do Maranhão. Assim, tendo em vista a problemática exposta, traz-se à seguinte questão norteadora do estudo: Quais os possíveis impactos da síndrome de burnout nos profissionais de um Serviço de Atendimento Móvel (SAMU) na cidade pesquisada, no interior do Maranhão?

\section{MÉTODOS}

Trata-se de uma pesquisa descritiva, exploratória de abordagem quantitativa. A pesquisa descritiva é descrever as características de determinadas populações ou fenômenos. Uma de suas peculiaridades está na utilização de técnicas padronizadas de coleta de dados, tais como questionários e a observação sistemática. A pesquisa foi realizada em uma instituição pública que realiza atendimentos de urgência emergência em um município do estado do Maranhão. Esta instituição é composta por uma equipe multiprofissional: enfermeiros, técnicos de enfermagem, auxiliares, médicos e motoristas, vigias, pessoal do serviço de limpeza e coordenação administrativa. Vale ressaltar que a escolha da instituição se deu por ser referência no estado de Maranhão, no atendimento pré-hospitalar, além de ser excelente campo aberto para as áreas de ensino, pesquisa e extensão ao cumprimento dos requisitos do estudo.

A população da pesquisa foi composta por profissionais que prestam atendimento pré-hospitalar e foram homens e mulheres que compõem o perfil profissional do objetivo do estudo, e que tenham de 20 anos ou mais de idade, com mais de 1 ano de serviço na instituição. Este estudo teve como critérios de inclusão todos os profissionais submetidos no plantão e que estivessem mais de um ano de desempenho das atividades assistenciais.

Já com relação aos critérios de exclusão selecionados foram todos os profissionais com menos de um ano de prestação de serviço no atendimento pré-hospitalar do Serviço de Atendimento Móvel da cidade pesquisada, no interior do Maranhão, os de férias, licença prêmio, licença médica e licença maternidade. Para esse estudo, a população utilizada foi um total de 58 profissionais. Após aplicação dos critérios de exclusão durante a coleta de dados, tendo como fator importante, identificar a quantidade correta de profissionais ainda atuantes no serviço de atendimento móvel de saúde. Foram excluídos: 9 profissionais por ter menos de 01 ano de atuação no serviço, 5 médicos, sendo 1 de licença sem vencimento, 1 por fazer parte do núcleo de ensino e os demais não encontrados nos plantões, 1 enfermeiro por não ser encontrado no plantão. $O$ estudo final teve uma população final de 43 profissionais (Tabela 1). 
Tabela 1 - Distribuição dos Profissionais da Saúde do Serviço de Atendimento Móvel de Urgência (SAMU) ( $n=43), 2020$.

\begin{tabular}{lc}
\hline Profissionais do SAMU & $(\mathbf{N})$ \\
\hline Médicos & 11 \\
Enfermeiros & 4 \\
Técnicos em enfermagem & 14 \\
Motoristas de ambulâncias & 14 \\
\hline Total & $\mathbf{4 3}$
\end{tabular}

Fonte: Cruz FMP, et al., 2020.

As variáveis deste estudo foram às que estavam relacionados com os aspectos à idade, sexo, estado civil, números de filhos, renda familiar, número de empregos, grau de formação, continuação dos estudos, função do cargo na instituição do estudo e turno que exerce, tempo de serviço.

É importante mencionar que instrumento utilizado nesta pesquisa foi elaborado pelas autoras por meio da aplicabilidade de questionários sociodemográficos e do autoaplicável enquanto que o Inventário de 24 avaliações do esgotamento profissional Maslach Burnout Inventory (MBI).

A classificação da escala é do tipo Liket que varia de zero a seis. É um questionário autoaplicável contendo 22 itens que avalia a síndrome de burnout por três dimensões. Ressalto ainda que a coleta de dados foi realizada no mês de fevereiro a março de 2019 e somente após aprovação do Comitê de Ética em Pesquisa da Plataforma Brasil (CEP) da Associação Piauiense de Combate ao Câncer/Hospital São Marcos com número da CAEE: 04938918.9.0000.5584 e sob o parecer ํㅜ: 3.131.983.

As análises dos dados obtidos no estudo foram analisadas e tabuladas estatisticamente por meio do Micro Excel 2016, utilizando o Programa Statistical Package for the Social Sciences (SPPS) versão 22. Análise descritiva é usada na descrição de dados por meios das variáveis, uso de números ou por medidas estatísticas que representam os dados da pesquisa. Análise inferencial é procedimento em estatística que é utilizada para formular conclusões e fazer inferências após a análise de dados coletados (RODRIGUES CFS, et al., 2017).

O teste estatístico utilizado foi o de Fisher serve para testar a hipótese no procedimento estatístico baseado em determinados parâmetros, com o objetivo de relacionar os resultados da escala de $\mathrm{SB} / \mathrm{MBI}$ com características sociodemográficos e aspectos profissionais.

\section{RESULTADOS}

Pode-se verificar no estudo que participaram 43 profissionais, sendo que a maioria dos profissionais 26 $(60,5 \%)$ eram do sexo masculino, a faixa etária predominantemente dos participantes esteve entre 31 a 50 anos $32(74,5 \%)$. No entanto, em relação ao estado civil a maioria corresponde $21(48,8 \%)$ aos casados, sendo que $22(51,2 \%)$ têm de 2 a 3 filhos. Já relacionado à formação acadêmica prevalente foi a de Ensino Médio com $14(32,6 \%)$ e $13(30,2 \%)$ de Ensino Superior, $34(79,1 \%)$ responderam que desejam continuar os estudos.

No que diz respeito à função exercida na instituição, a que mais prevaleceu foi a de técnica em enfermagem e motorista; ambos com 14 (32,6\%). Quanto aos turnos dos profissionais desta instituição percebe-se que $20(46,6 \%)$ trabalham no turno noturno e $8(18,6 \%)$ trabalham nos turnos diurno e noturno. Vale destacar que dos profissionais, $16(37,2 \%)$ afirmam trabalharem em dois empregos, o tempo de serviços com maior resposta foi o de 5 a 10 anos com $18(41,9 \%)$ respondido e $20(48,8 \%)$ afirmam receberem até dois salários mínimos (Tabela 2). 
Tabela 2 - Análise descritiva do perfil sociodemográfico de profissionais de saúde do Serviço de Atendimento Móvel de Urgência (SAMU) ( $\mathrm{n}=43), 2020$.

\begin{tabular}{|c|c|c|}
\hline Variáveis & (n) & $\%$ \\
\hline \multicolumn{3}{|l|}{ Sexo } \\
\hline Masculino & 26 & 60,5 \\
\hline Feminino & 17 & 39,5 \\
\hline \multicolumn{3}{|l|}{ Faixa etária } \\
\hline 22 a 30 anos & 5 & 11,6 \\
\hline 31 a 40 anos & 14 & 32,6 \\
\hline 41 a 50 anos & 18 & 41,9 \\
\hline$>51$ anos & 6 & 14,0 \\
\hline \multicolumn{3}{|l|}{ Estado civil } \\
\hline Solteiro (a) & 12 & 27,9 \\
\hline Casado (a) & 21 & 48,8 \\
\hline União estável & 3 & 7,0 \\
\hline Divorciado (a) & 6 & 14,0 \\
\hline Viúvo & 1 & 2,3 \\
\hline \multicolumn{3}{|l|}{ Filhos } \\
\hline 0 & 13 & 30,2 \\
\hline 1filho & 22 & 51,2 \\
\hline 2 a 3 filhos & 5 & 11,6 \\
\hline$>3$ filhos & 3 & 7,0 \\
\hline \multicolumn{3}{|l|}{ Formação acadêmica } \\
\hline Ensino médio & 14 & 32,6 \\
\hline Técnico & 7 & 16,3 \\
\hline Superior & 13 & 30,2 \\
\hline Pós-graduação & 9 & 20,9 \\
\hline \multicolumn{3}{|l|}{ Continuação dos estudos } \\
\hline Sim & 34 & 79,1 \\
\hline Não & 9 & 20,9 \\
\hline \multicolumn{3}{|l|}{ Função no SAMU } \\
\hline Enfermeiro & 4 & 9,3 \\
\hline Médico & 11 & 25,6 \\
\hline Téc. em enfermagem & 14 & 32,6 \\
\hline Motorista & 14 & 32,6 \\
\hline \multicolumn{3}{|l|}{ Turno de trabalho } \\
\hline Diurno & 15 & 34,9 \\
\hline Noturno & 20 & 46,5 \\
\hline Diurno-Noturno & 8 & 18,6 \\
\hline \multicolumn{3}{|l|}{ Vínculo de trabalho } \\
\hline Somente neste emprego & 15 & 34,9 \\
\hline Em dois empregos & 20 & 46,5 \\
\hline Mais de dois empregos & 8 & 18,6 \\
\hline \multicolumn{3}{|l|}{ Tempo de serviço } \\
\hline 1 a 3 anos & 5 & 11,6 \\
\hline 3 a 5 anos & 8 & 18,6 \\
\hline 5 a 10 anos & 18 & 41,9 \\
\hline 11 a 15 anos & 4 & 9,3 \\
\hline 6 a 20 anos & 5 & 11,6 \\
\hline$>21$ anos & 3 & 77,0 \\
\hline \multicolumn{3}{|l|}{ Renda mensal } \\
\hline Até 2 salários & 20 & 48,8 \\
\hline Acima de 3 salários & 7 & 17,1 \\
\hline 4 a 6 salários & 3 & 7,3 \\
\hline Acima de 6 salários & 10 & 24,4 \\
\hline Entre 2 e 3 salários & 1 & 2,4 \\
\hline
\end{tabular}

Fonte: Cruz FMP, et al., 2020 
Uma análise que permite a descrição dos profissionais baseados em questionamentos como o conhecimento de SB, sendo $27(65,9 \%)$ afirmam saberem sobre a mesma, $25(59,5 \%)$ disseram que às vezes se sentem esgotados fisicamente e mentalmente, mas apenas $4(10,5 \%)$ relatam sempre 28 fazerem algum tipo de exercício de relaxamento para controlar os sintomas que ocasionam o desgaste físico e mental. Somente $1(2,4 \%)$ informou fazer uso de medicação antidepressivo enquanto, $39(90,7 \%)$ responderam que nunca fizeram um acompanhamento psicoterápico (Tabela 3).

Tabela 3 - Análise descritiva do questionário autoaplicável respondidos por profissionais de saúde do Serviço de Atendimento Móvel de Urgência (SAMU) ( $n=43), 2020$.

\begin{tabular}{|c|c|c|}
\hline Conhecimento da Síndrome de Burnout & (n) & $\%$ \\
\hline Sim & 27 & 65,9 \\
\hline Não & 14 & 34,1 \\
\hline \multicolumn{3}{|l|}{ Esgotamento físico ou mental } \\
\hline Sempre & 4 & 9,5 \\
\hline Às vezes & 25 & 59,5 \\
\hline Nunca & 13 & 31,0 \\
\hline \multicolumn{3}{|c|}{ Ausência do trabalho por desgaste físico ou mental } \\
\hline Às vezes & 8 & 18,6 \\
\hline Nunca & 35 & 81,4 \\
\hline \multicolumn{3}{|l|}{ Irritação ou agressividade } \\
\hline Sempre & 1 & 2,4 \\
\hline Às vezes & 17 & 40,5 \\
\hline Nunca & 24 & 57,1 \\
\hline \multicolumn{3}{|l|}{ Mudanças de humor } \\
\hline Sempre & 2 & 4,8 \\
\hline Às vezes & 20 & 47, \\
\hline Nunca & 20 & 47 \\
\hline \multicolumn{3}{|l|}{ Exercício de relaxamento } \\
\hline Sempre & 4 & 10,5 \\
\hline Às vezes & 19 & 50,0 \\
\hline Nunca & 15 & 39,5 \\
\hline \multicolumn{3}{|l|}{ Atividade física } \\
\hline Sempre & 16 & 37,2 \\
\hline Às vezes & 21 & 48,8 \\
\hline Nunca & 6 & 14,0 \\
\hline \multicolumn{3}{|l|}{ Faz uso de ansiolítico } \\
\hline Não & 43 & 100 \\
\hline \multicolumn{3}{|l|}{ Faz uso de antidepressivo } \\
\hline Sim & 1 & 2,3 \\
\hline Não & 42 & 97,7 \\
\hline \multicolumn{3}{|l|}{ Psicoterapia } \\
\hline Sempre & 2 & 4,7 \\
\hline Às vezes & 2 & 4,7 \\
\hline Nunca & 39 & 90,7 \\
\hline
\end{tabular}

Fonte: Cruz FMP, et al., 2020

Mostram-se os principais sintomas que os entrevistados vêm apresentando ao longo do tempo, sendo o cansaço com $26,39 \%$ o que mais acomete os profissionais e em seguida os de dores musculares, com $25 \%$ da população do estudo (Gráfico 1). 
Gráfico 1 - Análise Descritiva dos sintomas presentes em profissionais de saúde do Serviço de Atendimento Móvel de Urgência (SAMU) ( $n=43), 2020$.

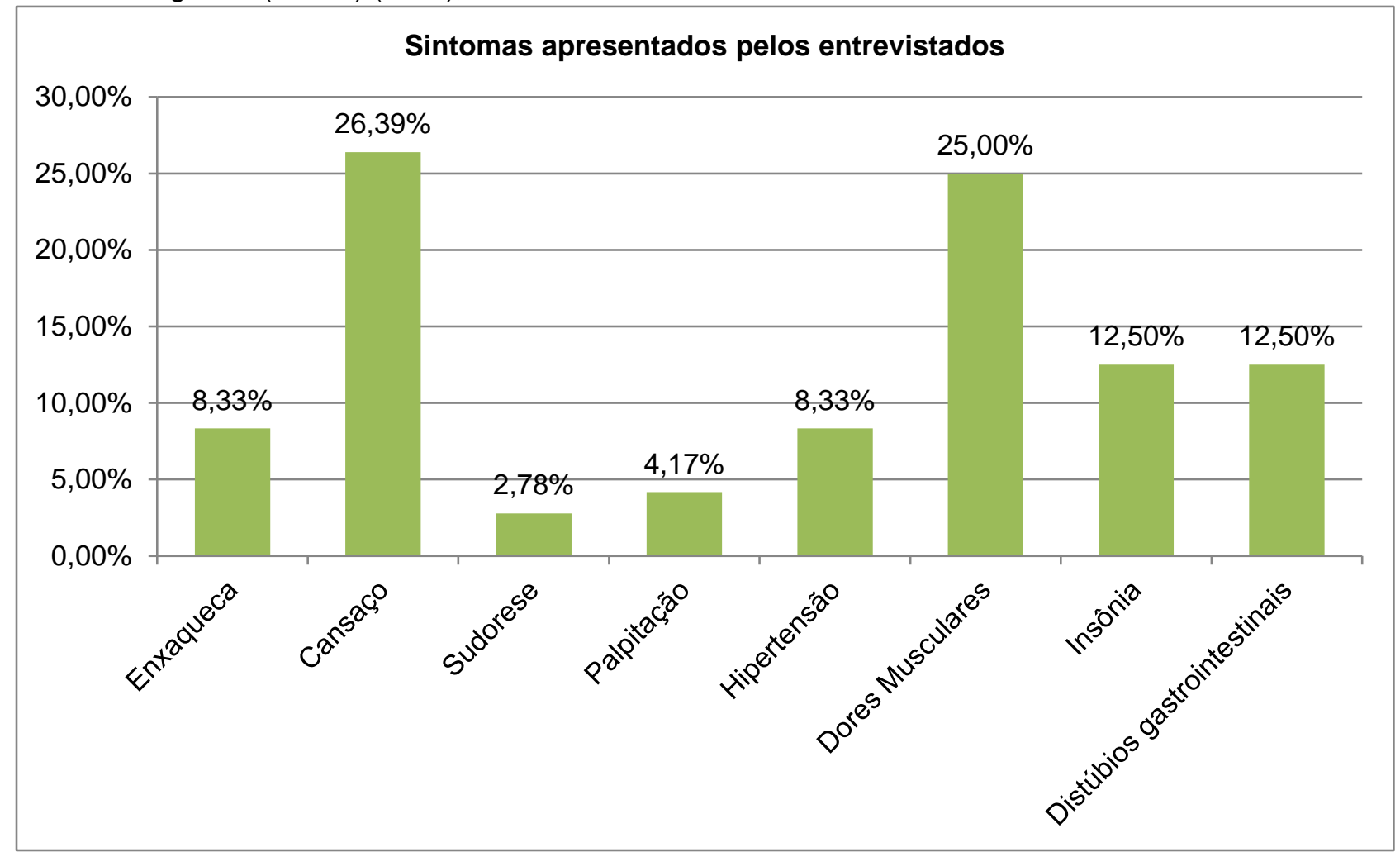

Fonte: Cruz FMP, et al., 2020.

Levou em consideração 22 perguntas da $\mathrm{MBI}$ com as respostas relacionadas às três dimensões da $\mathrm{SB}$, a exaustão emocional ( 9 itens), a despersonalização (5 itens) e baixa realização pessoal (8 itens). Todos os itens têm uma variância de respostas de 0 a 6 , sendo 0 (nunca), 1 (uma vez ao ano ou menos), 2 (uma vez por mês ou menos), 3 (algumas vezes por mês), 4 (uma vez por semana), 5 (algumas vezes por semana) e 6 (todos os dias) (NOGUEIRA LS, et al., 2018).

No que se refere à dimensão exaustão emocional $58,5 \%$ responderam nunca sentirem emocionalmente exausto no trabalho enquanto, $52,4 \%$ nunca sentiram estar trabalhando demais, nem que trabalhar com pessoas o dia todo exige grande esforço, sendo nunca um total de $56,1 \%$. Com isso, $56,4 \%$ disseram nunca, quando questionados devido ao trabalho exercido se estão insensíveis com as pessoas.

Quanto à despersonalização destacam-se os $61,0 \%$ que mencionaram sempre se sentirem cheios de alegrias e $62,5 \%$ sempre entendem com facilidades os sentimentos dos pacientes atendidos. Na realização pessoal observou-se que $71,4 \%$ nunca se sentiram frustrados com o trabalho, apenas $2,4 \%$ sempre se sentem cansados quando levantam e precisam encarar mais um dia de trabalho e já dos $90 \%$ confirmam nunca sentirem os pacientes como objetos (Tabela 4). 
Tabela 4 - Análise Descritiva da Classificação do Inventário de Avaliação do Esgotamento Profissional - Maslach Burnout Inventory (MBI) - respondidos por profissionais de saúde do Serviço de Atendimento Móvel de Urgência (SAMU) ( $n=43), 2020$.

\begin{tabular}{|c|c|c|c|c|c|c|c|}
\hline Grupos & $\begin{array}{c}\text { Nunca } \\
(\%)\end{array}$ & $\begin{array}{l}\text { Uma vez ao } \\
\text { ano ou menos } \\
(\%)\end{array}$ & $\begin{array}{l}\text { Uma vez por } \\
\text { mês ou menos } \\
(\%)\end{array}$ & $\begin{array}{l}\text { Algumas } \\
\text { vezes por mês } \\
(\%)\end{array}$ & $\begin{array}{l}\text { Uma vez por } \\
\text { semana }(\%)\end{array}$ & $\begin{array}{l}\text { Algumas vezes } \\
\text { por semana } \\
\text { Todos }(\%)\end{array}$ & $\begin{array}{l}\text { Todos os dias } \\
(\%)\end{array}$ \\
\hline \multicolumn{8}{|l|}{ Exaustão emocional } \\
\hline Sinto-me esgotado (a) ao final de um dia de trabalho. & $24,4 \%$ & $9,8 \%$ & $24,4 \%$ & $14,6 \%$ & $19,5 \%$ & $4,9 \%$ & $2,4 \%$ \\
\hline Sinto-me como se estivesse no meu limite. & $58,5 \%$ & $12,2 \%$ & $9,8 \%$ & $9,8 \%$ & $7,3 \%$ & $0,0 \%$ & $2,4 \%$ \\
\hline Sinto-me emocionalmente exausto/a com o meu trabalho. & $58,5 \%$ & $14,6 \%$ & $4,9 \%$ & $9,8 \%$ & $4,9 \%$ & $2,4 \%$ & $4,9 \%$ \\
\hline Sinto que estou trabalhando demais neste emprego. & $52,4 \%$ & $9,5 \%$ & $16,7 \%$ & $2,4 \%$ & $7,1 \%$ & $7,1 \%$ & $4,8 \%$ \\
\hline \multirow{2}{*}{$\begin{array}{l}\text { Trabalhar com pessoas o dia todo me exige um grande esforço. } \\
\text { Sinto que influencio positivamente a vida dos outros dos outros } \\
\text { através do meu trabalho. }\end{array}$} & $56,1 \%$ & $9,8 \%$ & $7,3 \%$ & $12,2 \%$ & $4,9 \%$ & $4,9 \%$ & $4,9 \%$ \\
\hline & $4,8 \%$ & $2,4 \%$ & $2,4 \%$ & $4,8 \%$ & $4,8 \%$ & $7,1 \%$ & $73,8 \%$ \\
\hline \multirow{2}{*}{$\begin{array}{l}\text { Lido de forma adequada com os problemas dos pacientes. } \\
\text { Sinto que sei tratar de forma tranquila os problemas emocionais } \\
\text { no meu trabalho. }\end{array}$} & $7,1 \%$ & $2,4 \%$ & $4,8 \%$ & $2,4 \%$ & $4,8 \%$ & $9,5 \%$ & $69,0 \%$ \\
\hline & $2,5 \%$ & $5,0 \%$ & $0,0 \%$ & $5,0 \%$ & $5,0 \%$ & $17,5 \%$ & $65,0 \%$ \\
\hline $\begin{array}{l}\text { Tenho me tornado mais insensível com as pessoas desde que } \\
\text { exerço este trabalho. }\end{array}$ & $56,4 \%$ & $10,3 \%$ & $12,8 \%$ & $7,7 \%$ & $2,6 \%$ & $0,0 \%$ & $10,3 \%$ \\
\hline \multicolumn{8}{|l|}{ Despersonalização } \\
\hline Sinto-me esgotado/ a com o meu trabalho. & $58,5 \%$ & $4,9 \%$ & $7,3 \%$ & $14,6 \%$ & $7,3 \%$ & $4,9 \%$ & $2,4 \%$ \\
\hline Sinto-me cheio/ a de alegria. & $2,4 \%$ & $2,4 \%$ & $4,9 \%$ & $9,8 \%$ & $2,4 \%$ & $17,1 \%$ & $61,0 \%$ \\
\hline \multirow{4}{*}{$\begin{array}{l}\text { Sinto-me estimulado/o depois de trabalhar em contato com os } \\
\text { pacientes. } \\
\text { Posso entender com facilidade o que sentem os pacientes. } \\
\text { Preocupa-me o fato de que este trabalho esteja me endurecendo } \\
\text { emocionalmente. } \\
\text { Sinto-me frustrado/ a com o meu trabalho. }\end{array}$} & $2,4 \%$ & $2,4 \%$ & $4,8 \%$ & $11,9 \%$ & $9,5 \%$ & $14,3 \%$ & $54,8 \%$ \\
\hline & $2,5 \%$ & $2,5 \%$ & $2,5 \%$ & $2,5 \%$ & $5,0 \%$ & $22,5 \%$ & $62,5 \%$ \\
\hline & $55,0 \%$ & $10,0 \%$ & $2,5 \%$ & $7,5 \%$ & $5,0 \%$ & $7,5 \%$ & $12,5 \%$ \\
\hline & $71,4 \%$ & $9,5 \%$ & $2,4 \%$ & $7,1 \%$ & $2,4 \%$ & $2,4 \%$ & $4,8 \%$ \\
\hline \multicolumn{8}{|l|}{ Baixa realização pessoal } \\
\hline $\begin{array}{l}\text { Trabalhar diretamente } \mathrm{cm} \text { pessoas me deixa muito estressado } \\
\text { (a). }\end{array}$ & $54,8 \%$ & $14,3 \%$ & $9,5 \%$ & $9,5 \%$ & $9,5 \%$ & $0,0 \%$ & $2,4 \%$ \\
\hline $\begin{array}{l}\text { Sinto-me cansado/a quando me levando de manhã e tenho que } \\
\text { encarar outro dia de trabalho. }\end{array}$ & $57,1 \%$ & $11,9 \%$ & $4,8 \%$ & $16,7 \%$ & $7,1 \%$ & $0,0 \%$ & $2,4 \%$ \\
\hline $\begin{array}{l}\text { Sinto que posso criar um ambiente tranquilo para os pacientes. } \\
\text { Tenho conseguido muitas realizações em minha profissão. }\end{array}$ & $\begin{array}{l}4,8 \% \\
2,5 \%\end{array}$ & $\begin{array}{r}4,8 \% \\
10,0 \%\end{array}$ & $\begin{array}{l}2,4 \% \\
0,0 \%\end{array}$ & $\begin{array}{l}9,5 \% \\
2,5 \%\end{array}$ & $\begin{array}{l}7,1 \% \\
5,0 \%\end{array}$ & $\begin{array}{l}11,9 \% \\
10.0 \%\end{array}$ & $\begin{array}{l}59,5 \% \\
70,0 \%\end{array}$ \\
\hline \multirow{3}{*}{$\begin{array}{l}\text { Sinto que os pacientes me culpam por alguns dos seus } \\
\text { problemas. } \\
\text { Sinto que os pacientes como se fossem objetos. } \\
\text { Não me preocupo realmente com o que ocorre com alguns dos } \\
\text { meus pacientes. }\end{array}$} & $76,9 \%$ & $7,7 \%$ & $0,0 \%$ & $2,6 \%$ & $2,6 \%$ & $5,1 \%$ & $5,1 \%$ \\
\hline & $90,0 \%$ & $5,0 \%$ & $2,5 \%$ & $0,0 \%$ & $2,5 \%$ & $0,0 \%$ & $0,0 \%$ \\
\hline & $70,0 \%$ & $5,0 \%$ & $2,5 \%$ & $2,5 \%$ & $2,5 \%$ & $5,0 \%$ & $12,5 \%$ \\
\hline
\end{tabular}

Fonte: Cruz FMP, et al., 2020. 


\section{DISCUSSÃO}

O Brasil ocupa uma área da América do Sul, com cerca de 200 milhões de habitantes, possui um sistema de saúde baseado na Universalidade, em 2002 pela portaria 2048 do Ministério da Saúde, regulamentou a Política Nacional de Atenção as Urgências em todo estado, com ênfase o Serviço de Atendimento Móvel de Urgência (SAMU), esse serviço conta com profissionais de ambos os sexos, além das categorias profissionais, médicos, equipe de enfermagem e motoristas das ambulâncias. Além disso, o serviço conta com características para o desenvolvimento de doenças ou consequências adquiridas no serviço (DUTRA AS, et al., 2019).

Quando comparado o sexo, no estudo de Souza AMJ et al. (2018), descreve que existe uma predominância do feminino com a SB, segundo o autor e seus colaboradores, pode-se relatar devido as várias responsabilidades envolvidas no sexo feminino, geralmente a mulher tem várias tarefas e compromissos. Ela é profissional, mãe, estudante e esposa, assimilando-se com os dados encontrados neste estudo.

De acordo como pesquisa referente à caracterização sócio demográfico de profissionais no SAMU da equipe de enfermagem, no estado do Paraná em 2017, citou que a média de idade dos profissionais do SAMU foi de 34,58 anos e a média do tempo de atuação no SAMU é de dois, quanto ao estado civil, nove (52,94\%) afirmaram serem solteiros, oito (47,06\%) eram casados. Já em relação à formação $11(64,71 \%)$ possuíam o Ensino Médio completo e seis (35, 29\%) ao Ensino Superior, para a renda mensal é em torno de 2 a 4 salários (RAMOS MA, et al., 2017). No que diz respeito ao item com relação ao ter filhos (76\%) declararam ter até 2 filhos (SANTOS JNMO, et al., 2019). Dados semelhantes foram encontrados neste estudo, apenas o número de filhos apresentou discreta diferença.

Worm FA et al. (2016) em sua pesquisa, evidenciou em relação ao estado civil dos enfermeiros da sua amostra, cerca de $(55,5 \%)$ eram casados e $(44,5 \%)$ eram solteiros. A faixa etária em sua maioria estava entre 20 aos 39 anos (89\%) e 11\% tinham mais de 40 anos. Nesta pesquisa foi possível observar que a maioria dos profissionais tinha a faixa etária menor que 41 anos, um dos atores que explica pode ser o fato da profissão de enfermagem estar crescendo nos últimos anos, aumentando a procura de pessoal mais jovem.

No estudo de Worm FA et al. (2016) para com o objetivo de mapear os fatores de risco para o desenvolvimento da Síndrome de Burnout em profissionais de enfermagem da Equipe do Serviço de Atendimento Móvel de Urgência, dos 18 participantes (78\%) eram técnicos e $(22 \%)$ enfermeiros, com a maioria do gênero feminino (77,78\%), com a faixa etária entre os 25 e 31 anos (33,33\%). (50\%) das participantes eram casadas e com filhos, o que corrobora com dados desta pesquisa.

Já em relação à idade associada ao profissional da medicina Rodrigues CFS (2017), aponta uma prevalência de 45 a 50 anos de idade, esse perfil pode ser um risco se associar a carga horária, ao horário de trabalho, ao tipo de paciente atendido nas ocorrências, às condições de trabalho e ainda a dificuldade de conciliar trabalho com a vida pessoal, e o afeto familiar.

Em um trabalho realizado por Pereira SS (2016) em Ribeirão Preto, Brasil em 2016 mostrou que em relação à formação por categoria, relevou que $49.6 \%$ eram técnicos de enfermagem, $20,9 \%$ seguido de enfermeiro, $11,3 \%$ e por fim médico $8,9 \%$. Quanto à relação ao cargo exercido, $42,9 \%$ eram técnicos de enfermagem, $16,9 \%$ eram enfermeiro $10,6 \%$ eram médicos e $2,1 \%$ outros. Sobre o turno de trabalho $77,7 \%$ é 42 diurno e 17,0\% noturno.

Quanto à atividade física 55\% disseram que não faz nenhuma atividade, em relação ao uso de psicofármacos, somente $13,8 \%$ fazem o uso, sobre o afastamento do trabalho $62,4 \%$ disseram nunca se afastaram. Segundo Dutra AS et al. (2019), afirma que o trabalho noturno pode desenvolver alterações fisiológicas e juntas impactar no bem-estar físico e psicológico dos profissionais, apresentando fadiga, baixo desempenho, insatisfação laboral e alterações no sono.

As características pessoais, tais como: idade, sexo, nível educacional, estado civil, ter filhos e personalidade não são por si mesmas desencadeantes do fenômeno, mas facilitadoras ou inibidoras da ação dos agentes estressores para SB (SEGUEL F, et al., 2018; RODRIGUES CFS, 2017). 
Ao avaliar o nível de estresse e conhecimento da SB entre os profissionais que atuam no SAMU é recomendado para a verificação da saúde dos profissionais, haja vista que, por meio de ações para manter o equilíbrio físico e psíquico dos profissionais, corroborando para reduzir fatores que compromete a qualidade de vida dessas pessoas (LUCCHESE R, et al., 2019; RAMOS MA, et al., 2017).

Os profissionais de enfermagem desenvolvem o seu desgaste emocional, na realização das suas atividades nos setores de assistência aos pacientes com câncer. Apresentam sentimentos, como: ansiedade, medos, impotência e também o peso do cuidado, onde a relação entre paciente e enfermeiro possibilita a ajuda no convívio. Entretanto, este convívio em vários momentos requer não somente o conhecimento técnico-científico, mas também apoio emocional, por serem momentos cheios de anseios, o que pode levar ao desgaste emocional nestes profissionais (KOLHS M, et al., 2016).

Resultado semelhante foi encontrado no estudo de Campos ICS et al. (2015), que mostrou que os enfermeiros e técnicos de enfermagem são profissionais com grande predisposição ao desenvolvimento desta síndrome, por serem os profissionais que ficam em contato por mais tempo ficam com o paciente e seus familiares. Além disto, no ambiente hospitalar, esses profissionais serem submetidos a péssimas condições de trabalho, que resultam em uma baixa qualidade de vida.

Na concepção de Pereira MMA e Gomes ARS (2016) os principais problemas que contribuem a síndrome de burnout, mostrou que os principais fatores de estresse, são as questões relacionadas ao cuidado com os clientes, a carreira, a baixa remuneração e o excesso da carga de trabalho, relacionados aos níveis de esgotamento e também a exaustão emocional, a despersonalização e os baixos índices de realização pessoal.

\section{CONCLUSÃO}

Portanto, esta pesquisa permitiu identificar os fatores nos profissionais que predominam para somatória da síndrome de burnout, como a idade, o tempo de serviço, a falta de atividade física ou de relaxamento para diminuir o nível de estresse, a responsabilidade da vida adulta, o estado civil e o próprio turno que trabalham. No que diz respeito à função exercida na instituição, os profissionais de enfermagem são os que mais sofrem com as pressões do trabalho e consequente desenvolvimento da Síndrome de Burnout, nota- se que profissionais casados, com filhos e com maior idade, são o grupo de risco para esta síndrome. Espera- se que este estudo subsidie ações de enfrentamento eficazes na prevenção e no combate a esta doença, bem como ações que estimulem o cuidado mental e meloria da qalidade de vida no trabalho.

\section{REFERÊNCIAS}

1. BATISTA LMA, et al. Síndrome de Burnout em enfermeiros do serviço de atendimento móvel de urgência. Temas em Saúde, João Pessoa, 2016; 16(3): 147-162.

2. CAMPOS ICS, et al. Fatores Sociodemográficos e Ocupacionais Associados à Síndrome de Burnout em Profissionais de Enfermagem. Psychology/Psicologia Reflexão e Crítica, 2015; 28(4): 764-771.

3. DUTRA FC, et al. The influence of medical work leaves in the perception of health and quality of life of adult individuals. Fisioterapia e Pesquisa. 2016; 23(1): 99-104.

4. DUTRA HS, et al. Burnout entre profissionais de enfermagem em hospitais no Brasil. Rev. CUID. 2019; 10(1): 1-13.

5. FERNANDES LS, et al. Associação entre síndrome de burnout, uso prejudicial de álcool e tabagismo na enfermagem nas UTIs de um hospital universitário. Rev. Ciências de Saúde Coletiva. 2018; 23(1): 203-214.

6. KOLHS M, et al. Sentimentos de Enfermeiros Frente ao Paciente Oncológico. J Health Sci, 2016; 18(4): $245-250$.

7. LUCCHESE R, et al. Transtorno mental comum entre indivíduos que abusam de álcool e drogas: estudo transversal. Texto Contexto Enferm, Goiás, 2017; 26(1): 1-07.

8. MORAIS AJD. Síndrome de Burnout em Médicos de Estratégia Saúde da Família de Montes Claros, MG, e Fatores Associados. Rev. Bras. Med. Fam. Comunidade. 2018; 13(40): 1-15.

9. MOTA GS, et al. Síndrome de Burnout em profissionais de saúde: uma revisão bibliográfica da literatura. REAS, Revista Eletrônica Acervo Saúde, Teresina- PI, 2016; 5(sup): 237-241.

10. NOGUEIRA LS, et al. Burnout and nursing work environment in public health institutions. Rev. bras. enfer. 2018; 71(2): 336-342.

11. OLIVEIRA LPS, ARAÚJO GF. Características da síndrome de Burnout em enfermeiros da emergência de um hospital público. Revista Enfermagem Contemporânea, 2016; 5(1): 34-42. 
12. PAIVA JDM, et al. Fatores desencadeantes da síndrome de burnout em enfermeiros. Rev. enferm. UFPE. 2019; 13(1): 483-490.

13. PEREIRA MMA, GOMES ARS. Stress, burnout e avaliação cognitiva: estudo na classe de Enfermagem. Arquivos Brasileiros de Psicologia, 2016; 68(1): 72-83.

14. PÊGO FPL, PÊGO DR. Síndrome de Burnout. Rev. Bras. Med. Trab. Anápolis/ GO, 2016; 14(2): 172-176.

15. RAMOS MA, et al. Concepção dos profissionais de serviço de emergência sobre qualidade de vida. Rev. ciên. biológicas e da saúde. 2017; 38(1): 35-44.

16. RODRIGUES CFS. Importância do uso adequado da estatística básica nas pesquisas clínicas. Rev. Bras anestesiol. 2017; 67(6): 619-625.

17. SANTOS JNMO, et al. Occupational Stress the Exposure of na Emergency Unit Nursing Team. Rev. Fund. Care. Online. 2019; 11(1): 455-463.

18. SARTORETO IS, KURGGANT P. Satisfação e Insatisfação no trabalho do enfermeiro. Rev. Bras. C. Saúde, 2017; 21(2): 181-188.

19. SEGUEL F, et al. A Síndrome de burnout em trabalhadores de enfermagem em dois hospitais no sul do Chile. Av Enferm, Chile, 2016; 34(1): 39-47.

20. SILVA CCS, et al. Burnout e tecnologias em saúde no contexto da enfermagem na Atenção Primária à Saúde. Esc Anna Nery, Rio Grande do Norte, 2017; 21(2): 1-7.

21. SILVA RAD, et al. Síndrome de Burnout. Fisioterapia e Pesquisa, 2018; 25(4): 388-394.

22. SILVA RNS, et al. Síndrome de burnout em profissionais da enfermagem. Saúde em foco, Teresina, 2015; 2(2): 94106.

23. SOUZA AMJ, et al. Síndrome de burnout: Fatores de risco em enfermeiros de unidades de terapia intensiva. Rev. Ciência \& Desenvolvimento, 2018;11(2): 23-34.

24. SOUSA GJ, ROSSI EJR. Síndrome de Burnout., Rev. Conexão Eletrônica Três Lagoas, 2016; 13(1): 1-06.

25. SOUSA MKP, et al. Síndrome de Burnout em profissionais de enfermagem. Revista eletronica Acervo Saúde. 2019; 5(supl): 12-23.

26. TAVARES JAB, et al. Os principais Fatores Geradores da Síndrome de Burnout no Enfermeiro. Rev. ESP. 2017; 11(1): 45-49.

27. VASCONCELOS EM, et al. Burnout and depressive symptoms in intensive care nurses: relationship analysis. Rev. Bras. Enferm. 2018; 71(1): 135-15.

28. WORM FA, et al. Risco de adoecimento dos profissionais de enfermagem no Trabalho em atendimento móvel de urgência. Rev Cuid, 2016; 7(2): 1288-96. 